\title{
The Usefulness of Dynamic $O$-(2-18 F-Fluoroethyl)-L-Tyrosine PET in the Clinical Evaluation of Brain Tumors in Children and Adolescents
}

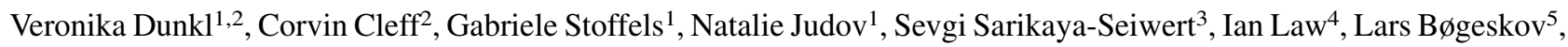 \\ Karsten Nysom ${ }^{6}$, Sofie B. Andersen ${ }^{4}$, Hans-Jakob Steiger ${ }^{3}$, Gereon R. Fink ${ }^{1,2}$, Guido Reifenberger ${ }^{7}$, Nadim J. Shah ${ }^{1}$, \\ Heinz H. Coenen ${ }^{1}$, Karl-Josef Langen ${ }^{1,8}$, and Norbert Galldiks ${ }^{1,2,9}$ \\ ${ }^{1}$ Institute of Neuroscience and Medicine, Research Center Jülich, Jülich, Germany; ${ }^{2}$ Department of Neurology, University of Cologne, \\ Cologne, Germany; ${ }^{3}$ Department of Neurosurgery, Heinrich Heine University Düsseldorf, Düsseldorf, Germany; ${ }^{4}$ Department of \\ Clinical Physiology, Nuclear Medicine and PET, Rigshospitalet, Copenhagen, Denmark; ${ }^{5}$ Department of Neurosurgery, Rigshospitalet, \\ Copenhagen, Denmark; ${ }^{6}$ Department of Pediatrics and Adolescent Medicine, Rigshospitalet, Copenhagen, Denmark; ${ }^{7}$ Department of \\ Neuropathology, Heinrich Heine University Düsseldorf, Düsseldorf, Germany; ${ }^{8}$ Department of Nuclear Medicine, University of Aachen, \\ Aachen, Germany; and ${ }^{9}$ Center of Integrated Oncology (CIO), University of Cologne, Cologne, Germany
}

Experience regarding $\mathrm{O}-\left(2-{ }^{18} \mathrm{~F}\right.$-fluoroethyl)-L-tyrosine $\left({ }^{18} \mathrm{~F}-\mathrm{FET}\right) \mathrm{PET}$ in children and adolescents with brain tumors is limited. Methods: Sixty-nine ${ }^{18}$ F-FET PET scans of 48 children and adolescents (median age, 13 y; range, 1-18 y) were analyzed retrospectively. Twenty-six scans to assess newly diagnosed cerebral lesions, 24 scans for diagnosing tumor progression or recurrence, 8 scans for monitoring of chemotherapy effects, and 11 scans for the detection of residual tumor after resection were obtained. Maximum and mean tumor-tobrain ratios (TBRs) were determined at 20-40 min after injection, and time-activity curves of ${ }^{18}$ F-FET uptake were assigned to 3 different patterns: constant increase; peak at greater than 20-40 min after injection, followed by a plateau; and early peak ( $\leq 20 \mathrm{~min}$ ), followed by a constant descent. The diagnostic accuracy of ${ }^{18} \mathrm{~F}$-FET PET was assessed by receiver-operating-characteristic curve analyses using histology or clinical course as a reference. Results: In patients with newly diagnosed cerebral lesions, the highest accuracy (77\%) to detect neoplastic tissue (19/26 patients) was obtained when the maximum TBR was 1.7 or greater (area under the curve, $0.80 \pm 0.09$; sensitivity, $79 \%$; specificity, $71 \%$; positive predictive value, $88 \%$; $P=0.02)$. For diagnosing tumor progression or recurrence, the highest accuracy $(82 \%)$ was obtained when curve patterns 2 or 3 were present (area under the curve, $0.80 \pm 0.11$; sensitivity, $75 \%$; specificity, $90 \%$; positive predictive value, $90 \%$; $P=0.02$ ). During chemotherapy, a decrease of TBRs was associated with a stable clinical course, and in 2 patients PET detected residual tumor after presumably complete tumor resection. Conclusion: Our findings suggest that ${ }^{18}$ F-FET PET can add valuable information for clinical decision making in pediatric brain tumor patients.

Key Words: glioma; kinetic pattern of ${ }^{18 \mathrm{~F} F-F E T ~ u p t a k e ; ~ m e t a b o l i c ~}$ imaging; contrast-enhanced MRI; children; FET PET

J Nucl Med 2015; 56:88-92

DOI: 10.2967/jnumed.114.148734

Received Sep. 17, 2014; revision accepted Nov. 13, 2014.

For correspondence or reprints contact: Norbert Galldiks, Institute of Neuroscience and Medicine, Research Center Jülich, 52425 Jülich, Germany. E-mail: n.galldiks@fz-juelich.de

Published online Dec. 18, 2014.

COPYRIGHT (c) 2015 by the Society of Nuclear Medicine and Molecular Imaging, Inc.
$\mathbf{P}$ most common causes of cancer-related death in children (1). The choice of antitumoral treatment depends on tumor histology, tumor location, and patient age, and management of central nervous system tumors in this patient group to date remains challenging. Brain tumors in children and adolescents comprise many tumor entities of varying malignancy—such as pilocytic astrocytoma, diffuse astrocytic gliomas including glioblastoma, ependymoma, medulloblastoma, and other central nervous system primitive neuroectodermal tumor types-as well as various other tumor types, for example, craniopharyngioma and germ cell tumors. Tumor location is heterogeneous, and in many cases, proximity to critical or eloquent brain structures precludes complete and partial resection and allows for diagnostic (stereotactic) biopsy only.

Concerning diagnostic imaging, contrast-enhanced structural MR imaging alone cannot always distinguish neoplastic from benign intracranial lesions. Moreover, the differentiation of treatment effects, such as radiation-induced changes, from recurrent or progressive disease may be difficult. In adult patients, PET using radiolabeled amino acids has been shown to improve diagnostic accuracy in patients with primary brain tumors, in particular gliomas $(2,3)$. In pediatric brain tumor patients (4-6), several studies suggest that amino acid PET with the tracer methyl- ${ }^{11} \mathrm{C}$ L-methionine ( $\left.{ }^{11} \mathrm{C}-\mathrm{MET}\right)$ may improve the management. Results of these studies also implicate that ${ }^{11} \mathrm{C}$-MET PET might be useful to differentiate neoplastic from nonneoplastic lesions in children and adolescents, in particular when a definite decision for further treatment based on a routine structural MR imaging procedures alone is difficult or impossible. Unfortunately, however, the use of ${ }^{11} \mathrm{C}-\mathrm{MET}$ is limited to PET centers with a cyclotron on site because of the short half-life of ${ }^{11} \mathrm{C}(20 \mathrm{~min})(7)$.

$O$ - $\left(2-{ }^{18} \mathrm{~F}\right.$-fluoroethyl)-L-tyrosine $\left({ }^{18} \mathrm{~F}-\mathrm{FET}\right)$ is a well-established ${ }^{18}$ F-labeled amino acid for PET (half-life, $109 \mathrm{~min}$ ) and offers logistic advantages over ${ }^{11} \mathrm{C}$-MET for clinical practice $(8,9)$. Investigations of adult brain tumor patients with PET using ${ }^{11} \mathrm{C}$-MET and ${ }^{18} \mathrm{~F}$-FET have reported comparable results for the 2 tracers (10). The evaluation of time-activity curves of ${ }^{18} \mathrm{~F}-\mathrm{FET}$ PET uptake may, however, provide valuable additional diagnostic 
information due to differential kinetic patterns of tracer uptake in high- and low-grade glioma (11-13), a phenomenon not observed with ${ }^{11} \mathrm{C}$-MET PET (14). For example, the kinetic pattern of a high-grade glioma appears to be characterized by an early peak after injection within the first $20 \mathrm{~min}$, followed by a decrease of ${ }^{18}$ F-FET uptake. In contrast, steadily increasing time-activity curves without identifiable peak of the tracer uptake are typical for low-grade gliomas $(12,13,15-19)$.

To date, experience with dynamic ${ }^{18}$ F-FET PET in children and adolescents with brain tumors is limited and is based on single case reports (20-22). Thus, the purpose of this study was to evaluate the diagnostic accuracy of this method in this particular age group.

\section{MATERIALS AND METHODS}

\section{Ethics}

This study represents a retrospective evaluation of a series of medically necessary ${ }^{18} \mathrm{~F}$-FET PET investigations in children and adolescents that were performed as compassionate use. The study was approved by the local ethics committee. There was no conflict with the Declaration of Helsinki. All investigations were performed on the expressed request and written documentation of the responsible pediatricians and in full agreement with the legal guardians. In all cases, there was no further diagnostic alternative except potentially lifethreatening invasive procedures. On the basis of the clinical experience in several thousand ${ }^{18} \mathrm{~F}$-FET PET investigations in adult patients without any side effects and the fact that no side effects or risks (except radiation burden) were to be expected because of the extremely low concentration of this nontoxic artificial amino acid, the risk of disease was judged as outweighing the risk of investigation.

\section{Patients}

Forty-eight children and adolescents 18 y or younger with brain tumors who had received dynamic ${ }^{18}$ F-FET PET scans at the Research Center Jülich and Rigshospitalet Copenhagen between 2006 and 2012 were identified retrospectively. All patients had been referred consecutively because decision making for further diagnostic procedures or treatment planning was difficult using the clinical presentation or MR imaging findings alone. The patients had been referred for ${ }^{18} \mathrm{~F}-\mathrm{FET}$ PET imaging for the assessment of newly diagnosed cerebral lesions ( $n=26$ scans in 26 patients), for diagnosis of possible tumor progression or recurrence of previously diagnosed brain tumors $(n=24$ scans in 18 patients), for monitoring of chemotherapy effects ( $n=8$ scans in 4 patients), and for the detection of residual tumor tissue after resection ( $n=11$ scans in 10 patients). Eight patients were examined for more than 1 indication. At the time of initial diagnosis, the median age of the patients was $13 \mathrm{y}$ (range, 1-18 y; 21 male and 28 female patients). The purpose of the scan and potential risks were explained to the patients and their legal guardians before they signed a document of informed consent before each ${ }^{18}$ F-FET PET examination as part of clinical routine. Sixty-nine ${ }^{18}$ F-FET PET scans were obtained for the entire cohort of patients $(n=48)$. In patients with newly diagnosed cerebral lesions, ${ }^{18} \mathrm{~F}$-FET PET was performed within the first $6 \mathrm{wk}$ after initial diagnosis. In patients with suspected tumor progression or recurrence, the time between initial diagnosis and ${ }^{18} \mathrm{~F}$-FET PET imaging was $24 \pm 35$ mo (range, 1-159 mo; median, $12 \mathrm{mo}$ ).

As reported before, the threshold of age to differentiate between children and adolescents was set at $15 \mathrm{y}$ (4). In the literature, a clear definition of an adolescent is not available; therefore, the threshold of $15 \mathrm{y}$ was set in the middle of the period of life between 10 and $20 \mathrm{y}$, which is the current definition of adolescence by the World Health Organization (WHO). At the time of ${ }^{18}$ F-FET PET imaging, 35 of the 48 patients $(73 \%)$ studied were younger than $15 \mathrm{y}$.

\section{PET Imaging with ${ }^{18} \mathrm{~F}$-FET and Data Analysis}

The amino acid ${ }^{18} \mathrm{~F}$-FET was produced via nucleophilic ${ }^{18} \mathrm{~F}$-fluorination with a specific radioactivity of greater than $200 \mathrm{GBq} / \mu \mathrm{mol}$ as described previously (23). The radiochemical yield of the tracer was about $60 \%-$ $65 \%$ at a radiochemical purity greater than $98 \%$. The tracer was administered as isotonic neutral solution. All patients had fasted for at least $6 \mathrm{~h}$ before PET scanning. Dynamic PET studies were acquired up to $50 \mathrm{~min}$ after intravenous injection of ${ }^{18} \mathrm{~F}-\mathrm{FET}$ on an ECAT EXACT HR+ scanner (Siemens Medical Systems, Inc.) (32 rings; axial field of view, $15.5 \mathrm{~cm}$ ) or on a Biograph Truepoint PET/CT scanner (Siemens) (4 rings; axial field of view, $21.8 \mathrm{~cm}$ ) in 3-dimensional mode. The dose of the injected radionuclide was adjusted for body weight (24). In addition, in children sedation or even general anesthesia, especially in very young children, was necessary to avoid patient movement. The emission recording consisted of at least 16 time frames covering the period up to $50 \mathrm{~min}$ after injection. For attenuation correction, transmission was measured with three ${ }^{68} \mathrm{Ge} /{ }^{68} \mathrm{Ga}$ rotating line sources or a CT-based attenuation correction was used. After correction for random and scattered coincidences and dead time, images were filtered with a gaussian filter of $5 \mathrm{~mm}$ in full width at half maximum. Image data were iteratively reconstructed (at least 40 image planes, 4 iterations and 12 subsets). The reconstructed image resolution was approximately $6.4 \mathrm{~mm}$. ${ }^{18} \mathrm{~F}$-FET uptake in the tissue was expressed as standardized uptake value (SUV) by dividing the radioactivity $(\mathrm{kBq} / \mathrm{mL})$ in the tissue by the radioactivity injected per gram of body weight.

${ }^{18}$ F-FET PET and, whenever available, contrast-enhanced MR imaging were coregistered using the MPI tool software (version 6.48; ATV). The fusion results were inspected and, if necessary, adapted based on anatomic landmarks. The regions-of-interest (ROI) analysis was based on the summed PET data from 20 to $40 \mathrm{~min}$ after injection (25). The transaxial slices showing the highest ${ }^{18} \mathrm{~F}$-FET accumulation in the tumors were chosen for ROI analyses. ${ }^{18} \mathrm{~F}$-FET uptake in the unaffected brain tissue was determined by a larger ROI placed on the contralateral hemisphere in an area of normal-appearing brain tissue including white and gray matter (25). ${ }^{18} \mathrm{~F}$-FET uptake in the tumor was determined with a 2-dimensional autocontouring process using a tumor-to-brain ratio (TBR) of 1.6 or greater. This cutoff is based on a biopsy-controlled study in cerebral gliomas in which a lesion-to-brain ratio of 1.6 separated best tumoral from peritumoral tissue (26). When ${ }^{18} \mathrm{~F}$-FET uptake of the lesion was similar to that of normal brain tissue, a representative irregular ROI was placed manually on the area of signal abnormality in the T1- and T2-weighted transversal MR scan and transferred to the coregistered ${ }^{18}$ F-FET PET scan (2).

Mean and maximum $\mathrm{TBRs}\left(\mathrm{TBR}_{\text {mean }}\right.$ and $\mathrm{TBR}_{\max }$, respectively) were calculated by dividing the mean and maximum SUV of the tumor ROI by the mean SUV of normal brain tissue in the ${ }^{18} \mathrm{~F}-\mathrm{FET}$ PET scan. Time-activity curves of mean SUV in the tumor and in the normal brain tissue were generated by applying these ROIs to the entire dynamic dataset. Time-to-peak (time in minutes from the beginning of the dynamic acquisition up to the maximum SUV of the lesion) was determined in the ROI of the tumor. As described previously (18), time-activity curves of each lesion were assigned to 1 of the following curve patterns: constantly increasing ${ }^{18} \mathrm{~F}$-FET uptake without identifiable peak uptake during data acquisition; ${ }^{18} \mathrm{~F}$-FET uptake peaking at a midway point $(>20-40 \mathrm{~min})$, followed by a plateau; and ${ }^{18}$ F-FET uptake peaking early ( $\left.\leq 20 \mathrm{~min}\right)$, followed by a constant descent (Fig. 1).

\section{Statistical Analysis}

Descriptive statistics are provided as mean and SD. To compare 2 different groups, the Student $t$ test for independent samples was used. The Mann-Whitney rank-sum test was used when variables were not distributed normally. $P$ values of less than 0.05 were considered significant. 


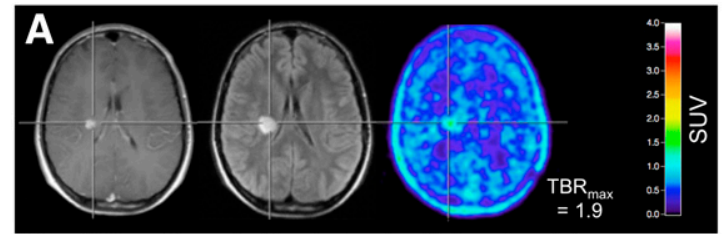

15 y-old boy, demyelinating lesion (multiple sclerosis)

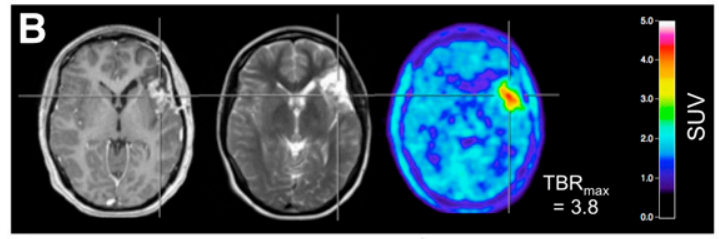

16 y-old girl, glioblastoma recurrence after $5 \mathrm{mo}$

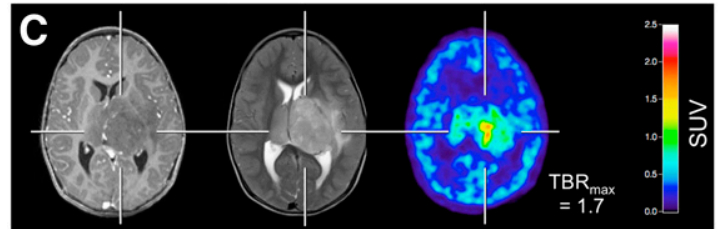

3 y-old boy, initial diagnosis of an anaplastic astrocytoma
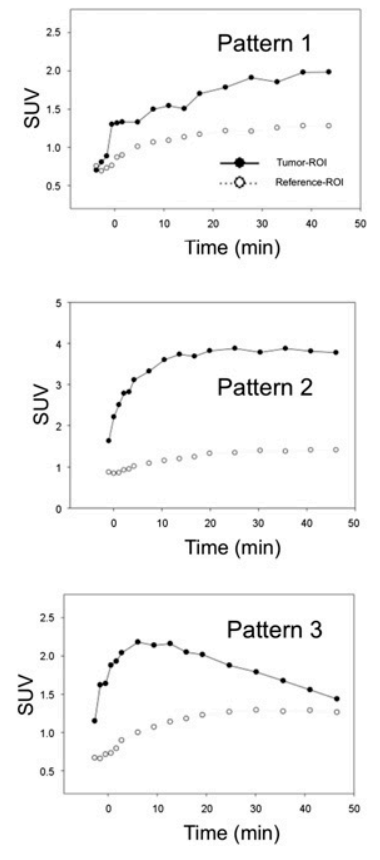

FIGURE 1. Patient examples with different kinetic patterns of ${ }^{18} \mathrm{~F}-\mathrm{FET}$ uptake. T1-weighted MR imaging on left, T2-/FLAIR-weighted MR imaging in middle, and ${ }^{18} \mathrm{~F}-\mathrm{FET}$ PET on right. (A) Patient with demyelinating lesion (i.e., multiple sclerosis) (patient 7) with moderately increased ${ }^{18} \mathrm{~F}-\mathrm{FET}$ uptake and curve pattern 1. (B) Recurrent glioblastoma (patient 26) with high ${ }^{18} \mathrm{~F}-\mathrm{FET}$ uptake and curve pattern 2. (C) Anaplastic astrocytoma (patient 10) with moderately increased ${ }^{18} \mathrm{~F}-\mathrm{FET}$ uptake and curve pattern 3 .

The diagnostic accuracy of the $\mathrm{TBR}_{\text {mean }}$ and $\mathrm{TBR}_{\max }$ of ${ }^{18} \mathrm{~F}-\mathrm{FET}$ uptake for differentiation of neoplastic lesions from nonneoplastic lesions in patients with newly diagnosed cerebral lesions and for differentiation of tumor progression or recurrence from unspecific posttherapeutic changes was evaluated by receiver-operating-characteristic curve analyses using histologic diagnosis or the clinical follow-up as a reference. The decision cutoff was considered optimal when the product of paired values for sensitivity and specificity reached its maximum. In addition, the area under the receiver-operating-characteristic curve, its SD, and the level of significance were determined as a measure of the diagnostic quality of the test. The diagnostic performance of curve patterns alone and in combination with TBRs was evaluated by the Fisher exact test for $2 \times 2$ contingency tables.

Statistical analysis was performed using SigmaPlot software (version 11.0; Systat Software Inc.) and PASW Statistics software (release 22.0.0; SPSS Inc.).

\section{RESULTS}

\section{Patients with Newly Diagnosed Cerebral Lesions}

In the 26 patients with newly diagnosed cerebral lesions, 26 PET scans were obtained. The diagnoses in the 26 patients with newly diagnosed cerebral lesions originally suggestive of neoplastic lesions/glioma were distributed as follows: WHO grade I dysembryoplastic neuroepithelial tumor $(n=1)$, WHO grade I pilocytic astrocytoma $(n=2)$, WHO grade II diffuse astrocytoma $(n=4)$, WHO grade III anaplastic astrocytoma $(n=4)$, WHO grade IV glioblastoma $(n=4)$, demyelinating lesion compatible with multiple sclerosis $(n=1)$, ischemic lesion/stroke $(n=1)$, arteriovenous vascular malformation $(n=1)$, low-grade glioma, WHO grade histologically not specified $(n=3)$, Rathke's cyst $(n=1)$, and hyperintense MR imaging lesions on
T2-/fluid-attenuated inversion recoveryweighted images without histologic confirmation $(n=4)$, one of which was confirmed as being due to a neoplastic lesion while 3 were proven as nonneoplastic lesions on follow-up (Supplemental Table 1; supplemental materials are available at http://jnm.snmjournals. org). Diagnoses were confirmed histologically in 20 of 26 patients $(77 \%)$. In 6 patients, the diagnosis of a brain tumor/ benign lesion was determined clinically (i.e., stable/unstable clinical course, follow-up MR imaging findings).

In this group of patients with newly diagnosed cerebral lesions, receiver-operatingcharacteristic analysis revealed that the highest accuracy $(77 \%)$ to detect neoplastic tissue (19/26 patients) was obtained when $\mathrm{TBR}_{\max }$ was 1.7 or greater (area under the curve, $0.80 \pm 0.09$; sensitivity, $79 \%$; specificity, $71 \%$; positive predictive value, $88 \% ; P=0.02$ ) (Supplemental Table 5). A corresponding patient example is presented in Figure 1A. The diagnostic accuracy was slightly lower when the $\mathrm{TBR}_{\text {mean }}$ was used as a parameter. The inclusion of kinetic parameters yielded no significant result regarding the differentiation of neoplastic from nonneoplastic lesions.

\section{Patients with Suspicion of Tumor Progression or Recurrence}

Eighteen patients with suspected tumor progression or recurrence underwent 24 PET examinations. The histologically confirmed initial diagnoses in patients with suspected tumor progression or recurrence $(n=18)$ were distributed as follows: WHO grade I pilocytic astrocytoma $(n=2)$, WHO grade II diffuse astrocytoma $(n=2)$, WHO grade III secondary anaplastic astrocytoma after malignant progression $(n=1)$, WHO grade II oligodendroglioma $(n=1)$, WHO grade III oligoastrocytoma $(n=1)$, WHO grade II ependymoma $(n=1)$, WHO grade III ependymoma $(n=2)$, WHO grade II pleomorphic xanthoastrocytoma $(n=1)$, WHO grade IV medulloblastoma $(n=1)$, WHO grade IV glioblastoma $(n=5)$, and malignant melanoma metastasis $(n=1)$. In 5 of these 18 patients, 2 or more PET scans were acquired (range of PET scans, 2-3). The diagnosis of presence or absence of tumor progression or recurrence was confirmed histologically in 5 of 18 patients (28\%; Supplemental Table 2). In 13 patients, the absence or presence of tumor progression or recurrence was determined clinically (i.e., stable/unstable clinical course, follow-up MR imaging findings, overall survival, treatment change) (Supplemental Table 2). In 13 of 24 cases, tumor progression or recurrence could be diagnosed.

For diagnosing tumor progression or recurrence, a high diagnostic accuracy was achieved when the $\mathrm{TBR}_{\text {mean }}$ and $\mathrm{TBR}_{\max }$ were used $(79 \%)$. The highest accuracy $(82 \%)$ was, however, obtained when the decision was based on kinetic parameters - that is, when curve patterns 2 or 3 were present (sensitivity, 75\%; specificity, 90\%; positive predictive value, $90 \% ; P=0.004$ ) (Supplemental Table 5). A corresponding patient example is presented in Figures $1 \mathrm{~B}$ and $1 \mathrm{C}$. 
Use of ${ }^{18}$ F-FET PET for Monitoring of Chemotherapy Effects

In 4 patients with WHO grade IV tumors, ${ }^{18}$ F-FET PET was used to monitor chemotherapy effects (Supplemental Table 3). Compared with baseline PET, in all patients a decrease of both $\mathrm{TBR}_{\max }$ (range of highest $\mathrm{TBR}_{\max }$ decrease, $21 \%-33 \%$ ) and $\mathrm{TBR}_{\text {mean }}$ (range of highest $\mathrm{TBR}_{\text {mean }}$ decrease, $10 \%-24 \%$ ) could be observed. During chemotherapy, a decrease of TBRs was associated with a stable clinical course for at least 6 mo. Additionally, in 3 of 4 patients a change of the kinetic pattern (from pattern 2 or 3 to pattern 1) and a prolongation of time-to-peak values (range, 15-35 min) were present. A complete response according to Macdonald criteria (27) as assessed by contrast-enhanced follow-up MR imaging could not be observed.

\section{Use of ${ }^{18}$ F-FET PET for Detection of Residual Tumor Tissue After Resection}

To detect residual tumor tissue after tumor resection, 10 patients were investigated by early postoperative MR imaging within the first $48 \mathrm{~h}$ and ${ }^{18}$ F-FET PET (median time after resection, $7 \mathrm{~d}$; range, 2-36 d) (Supplemental Table 4). One patient (patient 29) underwent surgery twice and was, therefore, examined twice. In 1 patient, the postoperative MR imaging, however, was not available (patient 16). Seven patients had high-grade tumors (WHO grade III or IV); the remaining 3 patients had low-grade tumors (WHO grade I or II). The early postoperative MR imaging yielded presence of contrast enhancement or nonenhancing tumor mass in 8 patients (considered as partial resection) and absence thereof in 1 patient (considered as complete resection). In patients with partial resection, ${ }^{18} \mathrm{~F}$-FET PET detected metabolically active tumor $\left(\mathrm{TBR}_{\max } \geq 1.7\right.$; Supplemental Table 4).

\section{DISCUSSION}

Our findings suggest that imaging parameters derived from ${ }^{18} \mathrm{~F}-$ FET PET may be helpful in pediatric patients with brain tumors, especially regarding the identification of newly diagnosed brain lesions suggestive of glioma and in the diagnosis of tumor progression or recurrence. The highest diagnostic accuracy (range, 73\%$77 \%$ ) was achieved by the use of a simple threshold-based ROI approach in newly diagnosed lesions and by the analysis of the kinetic pattern of ${ }^{18} \mathrm{~F}$-FET uptake in the diagnosis of possible tumor progression or recurrence $(82 \%)$. To the best of our knowledge, the present study evaluates for the first time dynamic parameters of ${ }^{18} \mathrm{~F}$-FET uptake in brain tumors of pediatric and adolescent patients and provides similar results as previously observed in adult glioma patients $(2,3)$.

Furthermore, first experiences in a small number of pediatric brain tumor patients in our study demonstrate that ${ }^{18}$ F-FET PET may be helpful for monitoring the effects of chemotherapy in malignant brain tumors. In most of these patients treated with chemotherapy, we observed a reduction of amino acid uptake. Our findings suggest that this finding is related to a stable clinical course. However, this observation should be reevaluated in a higher number of pediatric patients with the same histology and chemotherapy. Moreover, ${ }^{18}$ F-FET PET appears to be helpful to determine the residual tumor volume after brain tumor resection and may serve as a valuable tool for optimal planning of the further treatment strategy, for example, decision for re-resection, adjuvant radiotherapy. The reliability of ${ }^{18} \mathrm{~F}$-FET PET to identify residual tumor after surgery has not yet been proven by a biopsy-controlled study but previous studies using ${ }^{11} \mathrm{C}$-MET PET have demonstrated that amino acid PET is quite useful for this purpose (28). Nevertheless, to confirm these findings further studies are warranted.

To date, only a few amino acid PET studies have focused on children and adolescents with brain tumors, predominantly using PET with ${ }^{11} \mathrm{C}$-MET $(4-6,29)$. Pirotte et al. provided evidence that
${ }^{11} \mathrm{C}$-MET PET improves the diagnostic yield of stereotactic brain biopsies and surgical management of brain tumors in children $(6$, 30,31). Consistent with this finding, a first study using stereotactic biopsy guided by metabolic imaging with ${ }^{18}$ F-FET PET enhanced the diagnostic yield in diffuse pediatric gliomas and disclosed unexpected hot spots (20). Despite these encouraging results, only a few experiences in children and adolescents with brain tumors using ${ }^{18}$ F-FET PET have been reported $(21,22)$. With regard to this important clinical issue, our findings suggest that valuable additional information can be derived from ${ }^{18} \mathrm{~F}$-FET PET, which presents a rapidly developing new imaging method due to the logistical advantages, compared with PET using ${ }^{11} \mathrm{C}$-MET.

In patients with newly diagnosed cerebral lesions suggestive of glioma $(n=26), 15$ of 19 brain tumors were correctly identified as neoplastic lesions. However, 2 of 7 benign lesions (i.e., an ischemic lesion and a demyelinating lesion) were wrongly classified as brain tumors-that is, false-positive. In adults, moderate ${ }^{18} \mathrm{~F}$-FET uptake has been reported previously in inflammatory brain lesions and in the vicinity of cerebral ischemia, brain abscesses, and cerebral hemorrhage, which appears to result from tracer uptake in reactive gliosis (32-36). Therefore, it seems likely that the evaluation of pediatric brain tumors by ${ }^{18}$ F-FET PET may be affected negatively in the same way.

Currently, the relevance for the clinical use of dynamic ${ }^{18} \mathrm{~F}-\mathrm{FET}$ PET is still a matter of debate. Dynamic ${ }^{18} \mathrm{~F}$-FET PET imaging requires longer acquisition times ( $50 \mathrm{vs.} 20 \mathrm{~min}$ ), reducing the number of patients who can be investigated with 1 synthesis or delivery of ${ }^{18} \mathrm{~F}-\mathrm{FET}$ and increasing the costs of the investigation in routine clinical practice. Furthermore, the shorter acquisition time is more comfortable for the patients and reduces motion artifacts and, in younger children, duration of sedation or anesthesia. On the other hand, in 18 patients with suspected tumor progression or recurrence 24 PET examinations were performed and the highest diagnostic accuracy $(82 \%)$ could be obtained by the evaluation of curve patterns.

Furthermore, the differentiation between high- and low-grade glioma using amino acid PET is difficult. In children, a considerable overlap of amino acid uptake has been observed in low-grade and high-grade tumors (4,5). Similar to glucose metabolism (37), amino acid uptake may be high in low-grade tumors such as pilocytic astrocytoma and ganglioglioma, whereas uptake may be relatively low in patients with medulloblastoma of WHO grade IV (5).

One may argue that the results of our study are based on a relatively small number of investigated patients with a large variety of distinct entities and that our results should hence be treated with caution and confirmed in a larger series of patients. However, the results of this study are based on 48 patients and 69 observations, which constitute to date the largest sample of children or adolescents with brain tumors studied using static and dynamic ${ }^{18}$ F-FET PET, and the results are promising. With this caveat in mind, we thus recommend the use ${ }^{18} \mathrm{~F}$ FET PET as an additional diagnostic tool in children and adolescents with brain tumors, especially when the diagnostic information derived from standard MR imaging is equivocal.

In the near future, the use of hybrid PET/MR imaging scanners may provide more comprehensive information. A first report of Garibotto et al. (21) supports the acquisition of both amino acid PET and MR imaging in a single session on a hybrid system in pediatric patients. Besides the valuable improvement of simultaneously acquired diagnostic information, especially in children, the use of this technology minimizes the patients' discomfort (e.g., only a single transport to the imaging facility is necessary, resulting also in a substantial reduction of scanning time, avoidance of an additional sedation or general anesthesia by an anesthesiologist) and 
helps to optimize coregistration of both imaging modalities. However, the optimal strategy of MR imaging-based attenuation correction has not been identified at this moment. The present standard is the Dixon water-fat segmentation method, which systematically ignores bone and metal implants (38). The consequences for the distribution of metabolic activity need to be studied in detail before the clinical use in pediatric neuroonocology can be endorsed.

\section{CONCLUSION}

In pediatric and adolescent patients with cerebral tumors, both standard and kinetic imaging parameters derived from ${ }^{18} \mathrm{~F}-\mathrm{FET}$ PET may add valuable information for clinical decision making, especially in the field of differential diagnosis of newly diagnosed brain lesions suggestive of glioma and in the identification of tumor progression or recurrence after specific neurooncologic treatment.

\section{DISCLOSURE}

The costs of publication of this article were defrayed in part by the payment of page charges. Therefore, and solely to indicate this fact, this article is hereby marked "advertisement" in accordance with 18 USC section 1734. The Brain Imaging Center West supported this work. Guido Reifenberger has received a research grant from Roche and honoraria for advisory boards from Roche, Merck-Serono, and Amgen. No other potential conflict of interest relevant to this article was reported.

\section{ACKNOWLEDGMENTS}

We thank Suzanne Schaden, Elisabeth Theelen, and Kornelia Frey for their gentle and compassionate care of the young patients and their parents as well as Johannes Ermert, Silke Grafmüller, Erika Wabbals, and Sascha Rehbein for radiosynthesis of ${ }^{18}$ F-FET.

\section{REFERENCES}

1. Pollack IF, Jakacki RI. Childhood brain tumors: epidemiology, current management and future directions. Nat Rev Neurol. 2011;7:495-506.

2. Rapp M, Heinzel A, Galldiks N, et al. Diagnostic performance of ${ }^{18}$ F-FET PET in newly diagnosed cerebral lesions suggestive of glioma. J Nucl Med. 2013;54:229-235.

3. Dunet V, Rossier C, Buck A, Stupp R, Prior JO. Performance of ${ }^{18}$ F-fluoro-ethyltyrosine $\left({ }^{18} \mathrm{~F}\right.$-FET) PET for the differential diagnosis of primary brain tumor: a systematic review and metaanalysis. J Nucl Med. 2012;53:207-214.

4. Galldiks N, Kracht LW, Berthold F, et al. $\left[{ }^{11} \mathrm{C}\right]-\mathrm{L}-$-methionine positron emission tomography in the management of children and young adults with brain tumors. J Neurooncol. 2010;96:231-239.

5. Utriainen M, Metsahonkala L, Salmi TT, et al. Metabolic characterization of childhood brain tumors: comparison of ${ }^{18} \mathrm{~F}$-fluorodeoxyglucose and ${ }^{11} \mathrm{C}$ methionine positron emission tomography. Cancer. 2002;95:1376-1386.

6. Pirotte B, Goldman S, Salzberg S, et al. Combined positron emission tomography and magnetic resonance imaging for the planning of stereotactic brain biopsies in children: experience in 9 cases. Pediatr Neurosurg. 2003;38:146-155.

7. Singhal T, Narayanan TK, Jain V, Mukherjee J, Mantil J. ${ }^{11} \mathrm{C}$-L-methionine positron emission tomography in the clinical management of cerebral gliomas. Mol Imaging Biol. 2008;10:1-18.

8. Langen KJ, Hamacher K, Weckesser M, et al. O-(2-[ $\left[{ }^{18} \mathrm{~F}\right]$ fluoroethyl)-L-tyrosine: uptake mechanisms and clinical applications. Nucl Med Biol. 2006;33:287-294.

9. Wester HJ, Herz M, Weber W, et al. Synthesis and radiopharmacology of $O$-(2$\left[{ }^{18}\right.$ F]fluoroethyl)-L-tyrosine for tumor imaging. J Nucl Med. 1999;40:205-212.

10. Grosu AL, Astner ST, Riedel E, et al. An interindividual comparison of O- $\left(2-\left[{ }^{18} \mathrm{~F}\right]\right.$ fluoroethyl)-L-tyrosine $\quad$ (FET)- and L-[methyl- $\left.{ }^{11} \mathrm{C}\right]$ methionine (MET)-PET in patients with brain gliomas and metastases. Int J Radiat Oncol Biol Phys. 2011;81:1049-1058.

11. Galldiks N, Stoffels G, Ruge MI, et al. Role of $O-\left(2-{ }^{18} \mathrm{~F}\right.$-fluoroethyl)-L-tyrosine PET as a diagnostic tool for detection of malignant progression in patients with low-grade glioma. J Nucl Med. 2013;54:2046-2054.
12. Kunz M, Thon N, Eigenbrod S, et al. Hot spots in dynamic ${ }^{18}$ FET-PET delineate malignant tumor parts within suspected WHO grade II gliomas. Neuro-oncol. 2011;13:307-316.

13. Jansen NL, Graute V, Armbruster L, et al. MRI-suspected low-grade glioma: is there a need to perform dynamic FET PET? Eur J Nucl Med Mol Imaging. 2012;39:1021-1029.

14. Moulin-Romsée G, D'Hondt E, de Groot T, et al. Non-invasive grading of brain tumours using dynamic amino acid PET imaging: does it work for ${ }^{11} \mathrm{C}$-methionine? Eur J Nucl Med Mol Imaging. 2007;34:2082-2087.

15. Pöpperl G, Kreth FW, Mehrkens JH, et al. FET PET for the evaluation of untreated gliomas: correlation of FET uptake and uptake kinetics with tumour grading. Eur J Nucl Med Mol Imaging. 2007;34:1933-1942.

16. Pöpperl G, Kreth FW, Herms J, et al. Analysis of ${ }^{18}$ F-FET PET for grading of recurrent gliomas: is evaluation of uptake kinetics superior to standard methods? J Nucl Med. 2006;47:393-403.

17. Weckesser M, Langen $\mathrm{KJ}$, Rickert $\mathrm{CH}$, et al. O- $\left(2-\left[{ }^{18} \mathrm{~F}\right]\right.$ fluorethyl)-L-tyrosine PET in the clinical evaluation of primary brain tumours. Eur J Nucl Med Mol Imaging. 2005;32:422-429.

18. Calcagni ML, Galli G, Giordano A, et al. Dynamic O-(2-[ $\left.{ }^{18} \mathrm{~F}\right]$ fluoroethyl)L-tyrosine (F-18 FET) PET for glioma grading: assessment of individual probability of malignancy. Clin Nucl Med. 2011;36:841-847.

19. Galldiks N, Rapp M, Stoffels G, et al. Response assessment of bevacizumab in patients with recurrent malignant glioma using $\left[{ }^{18} \mathrm{~F}\right]$ fluoroethyl-L-tyrosine PET in comparison to MRI. Eur J Nucl Med Mol Imaging. 2013;40:22-33.

20. Messing-Jünger AM, Floeth FW, Pauleit D, et al. Multimodal target point assessment for stereotactic biopsy in children with diffuse bithalamic astrocytomas. Childs Nerv Syst. 2002;18:445-449.

21. Garibotto V, Heinzer S, Vulliemoz S, et al. Clinical applications of hybrid PET/ MRI in neuroimaging. Clin Nucl Med. 2013;38:e13-e18.

22. Korchi AM, Garibotto V, Ansari M, Merlini L. Pseudoprogression after proton beam irradiation for a choroid plexus carcinoma in pediatric patient: MRI and PET imaging patterns. Childs Nerv Syst. 2013;29:509-512.

23. Hamacher K, Coenen HH. Efficient routine production of the ${ }^{18} \mathrm{~F}$-labelled amino acid O-2- ${ }^{18} \mathrm{~F}$ fluoroethyl-L-tyrosine. Appl Radiat Isot. 2002;57:853-856.

24. Stauss J, Franzius C, Pfluger T, et al. Guidelines for ${ }^{18} \mathrm{~F}-\mathrm{FDG}$ PET and PET-CT imaging in paediatric oncology. Eur J Nucl Med Mol Imaging. 2008;35:1581-1588.

25. Langen KJ, Bartenstein P, Boecker H, et al. German guidelines for brain tumour imaging by PET and SPECT using labelled amino acids [in German]. Nuklearmedizin. 2011;50:167-173.

26. Pauleit D, Floeth F, Hamacher K, et al. O-(2-[ $\left.{ }^{18} \mathrm{~F}\right]$ fluoroethyl)-L-tyrosine PET combined with MRI improves the diagnostic assessment of cerebral gliomas. Brain. 2005;128:678-687.

27. Macdonald DR, Cascino TL, Schold SC Jr, Cairncross JG. Response criteria for phase II studies of supratentorial malignant glioma. J Clin Oncol. 1990;8:1277-1280.

28. Pirotte B, Goldman S, Van Bogaert $\mathrm{P}$, et al. Integration of $\left[{ }^{11} \mathrm{C}\right]$ methionine-positron emission tomographic and magnetic resonance imaging for image-guided surgical resection of infiltrative low-grade brain tumors in children. Neurosurgery. 2005;57:128-139.

29. O'Tuama LA, Phillips PC, Strauss LC, et al. Two-phase $\left[{ }^{11} \mathrm{C}\right] \mathrm{L}-\mathrm{methionine}$ PET in childhood brain tumors. Pediatr Neurol. 1990;6:163-170.

30. Pirotte B, Goldman S, Dewitte O, et al. Integrated positron emission tomography and magnetic resonance imaging-guided resection of brain tumors: a report of 103 consecutive procedures. J Neurosurg. 2006;104:238-253.

31. Pirotte B, Acerbi F, Lubansu A, Goldman S, Brotchi J, Levivier M. PET imaging in the surgical management of pediatric brain tumors. Childs Nerv Syst. 2007;23:739-751.

32. Salber D, Stoffels G, Pauleit D, et al. Differential uptake of $\left[{ }^{18} \mathrm{~F}\right]$ FET and $\left[{ }^{3} \mathrm{H}\right] 1-$ methionine in focal cortical ischemia. Nucl Med Biol. 2006;33:1029-1035.

33. Salber D, Stoffels G, Pauleit D, et al. Differential uptake of $O-\left(2-{ }^{18} \mathrm{~F}\right.$-fluoroethyl)L-tyrosine, L- ${ }^{3} \mathrm{H}$-methionine, and ${ }^{3} \mathrm{H}$-deoxyglucose in brain abscesses. $\mathrm{J} \mathrm{Nucl}$ Med. 2007;48:2056-2062.

34. Salber D, Stoffels G, Oros-Peusquens AM, et al. Comparison of $O-\left(2-{ }^{18} \mathrm{~F}\right.$-fluoroethyl)L-tyrosine and $\mathrm{L}^{-3} \mathrm{H}$-methionine uptake in cerebral hematomas. $\mathrm{J}$ Nucl Med. 2010;51:790-797.

35. Floeth FW, Pauleit D, Sabel M, et al. ${ }^{18}$ F-FET PET differentiation of ringenhancing brain lesions. J Nucl Med. 2006;47:776-782.

36. Hutterer M, Nowosielski M, Putzer D, et al. $\left[{ }^{18} \mathrm{~F}\right]$-fluoro-ethyl-L-tyrosine PET: a valuable diagnostic tool in neuro-oncology, but not all that glitters is glioma. Neuro-oncol. 2013;15:341-351.

37. Weckesser M, Matheja P, Rickert CH, et al. High uptake of L-3-[ $\left.{ }^{123} \Gamma\right]$ iodo-alpha-methyl tyrosine in pilocytic astrocytomas. Eur J Nucl Med. 2001;28:273-281.

38. Andersen FL, Ladefoged CN, Beyer T, et al. Combined PET/MR imaging in neurology: MR-based attenuation correction implies a strong spatial bias when ignoring bone. Neuroimage. 2014;84:206-216. 